Revue d'histoire de l'Amérique française

AST REVUE D.HISTOIRE DE L'AMÉRIQUE FRANÇAISE

\title{
La vie traditionnelle sur la côte de Beaupré, au début du $\mathrm{XX}^{\mathrm{e}}$ siècle
}

\section{Louis-Jacques Dorais}

Volume 19, numéro 4, mars 1966

URI : https://id.erudit.org/iderudit/302511ar

DOI : https://doi.org/10.7202/302511ar

Aller au sommaire du numéro

Éditeur(s)

Institut d'histoire de l'Amérique française

ISSN

0035-2357 (imprimé)

1492-1383 (numérique)

Découvrir la revue

Citer cet article

Dorais, L.-J. (1966). La vie traditionnelle sur la côte de Beaupré, au début du XX siècle. Revue d'histoire de l'Amérique française, 19(4), 535-550.

https://doi.org/10.7202/302511ar d'utilisation que vous pouvez consulter en ligne.

https://apropos.erudit.org/fr/usagers/politique-dutilisation/ 


\section{LA VIE TRADITIONNELLE SUR LA COTE DE BEAUPRÉ, AU DEEBUT DU XX' SIËCLE *}

L'auteur de cet article est étudiant en anthropologie à l'Université de Montréal et s'intéresse particulièrement à l'ethnographie du Canada français. Il reconnaît n'avoir pas écrit une étude "très approfondie". Mais il s'est renseigné à bonnes sources, "auprès d'informateurs qui ont toujours vécu sur la côte de Beaupré" et qui en étaient aux environs de la vingtaine à l'époque où se situe ce qu'ils ont raconté à l'auteur. Mode de vie aujourd'hui disparu, mais dont il convient de recueillir les vestiges.

Dans certaines régions rurales du Québec, les manières de vivre et les coutumes introduites sous le régime français se sont conservées presque intégralement jusqu'au début du $\mathrm{XX}^{\bullet}$ siècle. C'est le cas de la côte de Beaupré. Vers 1925, on pouvait encore y trouver une structure économique, sociale et culturelle qui remontait au temps de la colonie. Dans les années qui suivirent, la région commença à s'industrialiser et rapidement, elle acquit le même rythme et le même genre de vie que n'importe quelle autre société contemporaine.

Dans les pages qui suivent, nous allons décrire la vie traditionnelle telle qu'on pouvait l'y observer dans le premier quart du $\mathrm{XX}^{\mathrm{e}}$ siècle, ainsi que le cadre matériel et culturel dans lequel s'inscrivait cette vie. Notre travail sera divisé en quatre parties. Nous étudierons d'abord la culture matérielle, c'est-à-dire tout ce qui est palpable, concret: nourriture, vêtements, outils, etc. Puis nous jetterons un coup d'œil sur la vie quotidienne et les relations sociales. Nous terminerons enfin par une brève description du folklore de la région.

Avant de commencer, rappelons que la côte de Beaupré est située sur la rive nord du Saint-Laurent, en aval de Québec et en face de l'île d'Orléans. Elle s'étend de la rivière Montmorency 
jusqu'à Sainte-Anne de Beaupré. A l'époque, elle comprend trois paroisses: L'Ange-Gardien, Château-Richer et Sainte-Anne. Le relief y est très marqué. Tout le long de la côte, le bouclier Laurentien se termine abruptement par une falaise. Entre le pied de celle-ci et le fleuve, il n'y a pas un mille. A L'AngeGardien (c'est sur cette localité que porte la majeure partie de nos observations) la plupart des fermes sont bâties au sommet ou à mi-pente du coteau. Un chemin de terre relie chacune d'elles à la rive du fleuve.

\section{I - CULTURE MATÉRIELLE}

Sur la côte de Beaupré, on rencontre à cette époque (et il en subsiste encore beaucoup) deux sortes de maisons: à toit en pente et à combles français. Ces deux types d'habitation diffèrent par la forme de la couverture. La manière dont on les a construittes et l'agencemenent des pièces sont généralement les mêmes.

L'habitation traditionnelle est en pierre. Elle a fort belle apparence avec son extérieur blanchi à la chaux. Sa solidité est à toute épreuve, l'épaisseur des murs pouvant atteindre trois ou quatre pieds. Le toit est supporté de l'intérieur par des poutres entrecroisées. Notons que ces poutres sont fixées par des chevilles de bois, aucun clou n'entrant dans la construction de la maison.

En général, seul le rez-de-chaussée est habité, la partie située sous les combles servant de grenier. Ce rez-de-chaussée se divise en quatre ou cinq pièces. Une grande cuisine de forme rectangulaire en occupe le centre. Deux chambres à coucher s'ouvrent sur un de ses côtés. L'autre côté donne sur l'extérieur de la maison. C'est là que se trouve la porte d'entrée. A l'une des extrémités de la cuisine, il y a un petit salon. Dans certaines maisons plus grandes que les autres, on peut ajouter à ces pièces une grande salle à tout faire et un boudoir (qu'on appellera "salle d'en arrière"). Souvent, on a converti une partie du grenier en cinq ou six chambres à coucher.

La cuisine est le centre de la maison. C'est la pièce où l'on se tient le plus souvent. Le meuble principal en est une grande 
table en planches, bordée de longs bancs de bois. En général, on y trouve aussi quatre chaises (dont deux berçantes). Ces chaises sont réservées aux parents et aux grands-parents. C'est dans la cuisine qu'est placé le poêle à deux ponts, qui sert aussi bien à cuire les repas qu'à chauffer la maison. Il faut ajouter à cela l'évier et sa pompe, la boîte à bois, le séparateur à lait. Souvent aussi, on y a mis un "bed" ou lit-banc.

Le mobilier des chambres à coucher est à peu près semblable dans toutes les maisons. Il consiste en un lit à quenouilles, un chiffonnier et une commode aux multiples tiroirs. Le lit est couvert d'une paillasse, de draps de lin et de couvertures de laine. Le salon ne sert que dans les grandes occasions. On y trouve une table et quelques chaises, parfois un piano. Quand l'habitation compte une grande salle, celle-ci est meublée comme le salon avec, en plus, un buffet où l'on range la lingerie et un petit poêle pour les jours froids. C'est dans le grenier qu'on range les divers appareils servant au travail domestique: métier à tisser, rouet, cannelier, ourdissoir, dévidoir, cardes, etc. En hiver, on descend ces appareils dans la cuisine ou la grande salle.

Le couvert de table est composé d'assiettes et de tasses en granit. Les jours de fête, on emploie un ensemble en pierre. Les ustensiles (couteaux, fourchettes, cuillères, chaudrons, etc.) sont en fer. Quand il faut aller chercher l'eau à l'extérieur de la maison, on possède un joug qu'on place sur ses épaules et aux extrémités duquel on accroche des seaux de fer blanc. Ce sont des lampes à l'huile qui éclairent la maison. Malgré cela, on a gardé les anciens moules à chandelles et chandeliers à globe, qui trouvent encore maintes occasions de servir. Sur la table du salon, il y a un encrier et une plume d'oie.

Les seules décorations apposées aux murs sont les photos de famille et les images saintes. Dans la cuisine, il y a une grande croix de bois noire (symbolisant la tempérance) et une statue du Sacré-Cœur devant laquelle brûle un lampion. A certaines périodes de l'année, on la remplace par une effigie de sainte Anne ou de Notre-Dame. Aux fenêtres, il y a des rideaux plissés, de la grandeur de la vitre. Dans le salon et les chambres 
à coucher, le plancher est recouvert de catalognes. Dans la cuisine, où rien ne le revêt, il est ordinairement peint en jaune orange.

Un peu à l'écart de la maison s'élèvent les bâtiments de la ferme. Le plus vaste d'entre eux est évidemment la grange, où on loge les bêtes à cornes et les chevaux et où l'on amasse le foin et le grain. Une des extrémités de la grange sert de remise à voitures et de glacière. On y conserve la viande dans des coffres remplis de foin et de glace. La plupart des cultivateurs possèdent aussi une porcherie, une bergerie et un poulailler. On fait boucherie de porc et de veau à Pâques et en automne. C'est en décembre qu'on tue le mouton.

Parmi les céréales cultivées, on peut citer le blé, l'avoine, l'orge et le maîs. Il faut ajouter à cela plusieurs légumes (nommes de terre, carottes, choux) et quelques fruits (pommes, prunes). Une partie de ces produits est consommée par la famille tandis que le reste est vendu au marché à Québec. Les semailles se font au printemps, à la main. La récolte de foin a lieu en juillet, celle de blé à l'Assomption.

Les instruments aratoires, assez nombreux, sont tous actionnés par des chevaux. Mentionnons la charrue (à laquelle, par exception, on attelle parfois des bœufs), la faucheuse, la râcleuse et la moissonneuse. On transporte le foin dans des charrettes. C'est au moulin public que le grain est transformé en farine. On l'y charroie dans des banaux (sortes de voitures à deux roues). Ce moulin, qui se dresse à Château-Richer, a été bâti au XVII siècle. Si on le voulait, sa machinerie, complètement en bois, fonctionnerait encore de nos jours. Ajoutons enfin que les clôtures qui bornent les champs sont faites de pieux.

Les vêtements sont fabriqués en étoffe du pays et en flanelle. Celle-ci peut être teinte de différentes couleurs (on achète les teintures à la ville). Les hommes portent des pantalons et une chemise carreautée. Les femmes sont vêtues de jupes et de robes en flanelle mince. Les sous-vêtements sont en toile. On se chausse de bas de laine et de souliers de bœuf lacés (sembla- 
bles à des mocassins) ou encore de "bottes sauvages" (toujours en peau de bœuf). Les souliers de cuir tanné ne sont portés que dans les grandes occasions. Ordinairement, il n'y en a qu'une ou deux paires, servant à toute la famille. En hiver, on se couvre d'un manteau épais en étoffe du pays. Les hommes portent la ceinture fléchée et le casque de poil. Les femmes se coiffent d'un châle de laine. Par-dessus les souliers, on passe une paire de gros bas côtelés et des couvre-chaussures. Les robes de nuit sont en flanellette grise.

Le menu, assez monotone sur semaine, devient plus varié les jours de fête. Ordinairement, le repas du matin se compose de crêpes. Le midi, on mange de la soupe (aux pois, aux légumes, aux fèves) et du lard salé. Le soir, c'est du hachis ou du ragoût, précédé d'une soupe et accompagné de légumes. Aux deux repas principaux, on a de la mélasse comme dessert. On boit du lait, du thé et une sorte de café fait avec des croûtes de pain. Le pain de ménage accompagne chaque repas. Le samedi, la maîtresse de maison fait des tartes à la "pichoune" (aux raisins) et des gâteaux qu'on mangera le lendemain comme dessert. En hiver, surtout pendant le temps des fêtes, il y a grande consommation de tourtières. A cette période de l'année, les réunions de famille et les veillées sont très nombreuses. La cuisine se diversifie: "plorines" (saucisse en coiffe), andouilles, boudin, cretons, tête fromagée et surtout une sorte de gros macaroni qu'on achète à Québec et qui est fort apprécié de tout le monde. On boit du whisky et du vin de pissenlits, de gadelles ou de cerises.

Nous terminerons cette description de la culture matérielle en disant un mot des moyens de transport. Celui qui veut se rendre à Québec a le choix entre le train, circulant au niveau du fleuve, et l'omnibus. Ce dernier est une longue voiture, tirée par six chevaux et pouvant transporter plusieurs passagers. Il emprunte le chemin royal qui passe sur le haut des côtes. Quand on a traversé le pont de la rivière Montmorency, du côté de Beauport, on doit s'arrêter à une barrière et payer un droit de passage de dix sous. Chaque ferme possède plusieurs voitures: 
"l'express", véhicule à deux roues portant deux sièges dos à dos ; la "waguine" à quatre roues; le "quatre poteaux" comportant de grandes roues, mais pas de siège. Il y a aussi de petites voitures auxquelles on attelle des chiens. En hiver, on se promène en carriole (un ou deux chevaux) et en "cotter", petit traîneau à deux places. Notons enfin que celui qui veut se rendre à l'île d'Orléans en été n'a qu'à y aller en voiture. Il lui suffit de descendre au bord du fleuve et de s'avancer dans l'eau. La voiture flotte, le cheval nage et on arrive à bon port.

\section{II - VIE QUOTIDIENNE}

Nous allons maintenant décrire brièvement l'emploi du temps chez les cultivateurs ainsi que leurs distractions. Sur semaine, le lever a lieu vers cinq heures du matin. Après avoir "fait le train" des animaux et avoir déjeuné, on conduit les vaches au champ. Toute la joưrnée est consacrée aux travaux agricoles (labourage, semailles, moisson selon la saison). La seule halte est constituée par le repas du midi. Vers cinq heures du soir, on rentre les bêtes et on les trait. Puis c'est le souper et, à sept heures, la prière. Après cela, on couche les enfants et les adultes veillent jusque vers neuf heures. En été, la clarté tardive permet de ne pas allumer la lampe. Pendant l'hiver, naturellement, il n'y a pas de travaux champêtres. Après le déjeuner du matin, les hommes montent dans la forêt pour couper du bois tandis que les femmes restent à la maison et travaillent au métier à tisser. Pour le reste, l'emploi du temps est le même.

Le dimanche, on se rend à l'église pour la grand'messe de neuf heures. Chacun apporte avec soi son repas du midi, car il faut rester au village pour la séance de catéchisme. Celle-ci commence vers une heure de l'après-midi et elle est suivie, à deux heures, par les vêpres. Vers quatre heures trente, tout le monde est revenu chez soi. La journée se termine comme sur semaine par le souper, la prière et le coucher.

Les distractions sont très simples. Le soir, en famille, on chante des chansons de folklore. Les grands-parents captent l'attention de leurs petits-enfants avec des contes de fée. Pen- 
dant la journée, les vieillards se réunissent pour jouer aux dames. Mais le grand événement hebdomadaire, c'est la veillée du samedi soir. D'une semaine à l'autre, il se trouve toujours quelqu'un pour inviter ses voisins à passer la soirée chez lui.

Dans toutes les veillées, on chante, on danse et on s'adonne à des jeux de société. Il est à noter que contrairement à d'autres régions du Québec on n'accorde pas (ou on n'accorde plus) beaucoup d'importance aux contes. Les hommes se racontent bien quelques "histoires", mais il n'y a pas de conteurs professionnels connaissant par cœur plusieurs récits et les racontant dans les veillées (en Gaspésie, il en existe encore de nos jours; consulter Carmen Roy: "Littérature orale en Gaspésie"). Les chansons les plus populaires sont les chansons "à répondre". Chacun possède son répertoire, les hommes comme les femmes. On ne chante que rarement un refrain qui ne nous appartient pas, qui est ordinairement chanté par quelqu'un d'autre.

Parmi les principales danses, on peut citer le caledonia, le menuet, le brandy, les quadrilles, la bistringue et les gigues, simples ou doubles. Tout le monde danse ensemble, les jeunes comme les vieux, au son du violon, de l'accordéon ou de la clarinette. Ordinairement, le violoneux offre ses services gratuitement. Il est prêt à jouer toute la nuit, pourvu qu'on lui donne à boire de temps en temps.

Comme jeux de société, mentionnons la chaise honteuse, les jeux d'assiette et le jeu de Colin-Maillard (qu'on appelle "Colimailla"). Entre les chants, les danses et les jeux, la conversation roule bon train. Les hommes s'assemblent dans la cuisine et les femmes dans la grande salle. Quand la maison ne compte pas de grande salle, les femmes se placent à une extrémité de la cuisine et les hommes à l'autre. Ainsi, on s'amuse ferme et quand arrive le temps de partir, chacun n'espère qu'une seule chose: que la prochaine veillée revienne le plus vite possible.

\section{III - RELATIONS SOCIALES}

La structure de la société est très homogène. Il n'y a pour ainsi dire pas de classes sociales. Les seules personnes qui de 
par leur fonction se distinguent de la masse des cultivateurs sont le curé, le médecin et le maire. Les deux premiers, à cause du prestige conféré par leur formation professionnelle, ont une grande influence sur la communauté. Les conseils du curé (même dans le domaine matériel) sont presque considérés comme des ordres. Mais cela n'empêche pas une certaine indépendance de la part des cultivateurs. Le maire, qui est un des leurs, est le porte-parole de leurs idées et de leurs initiatives. Les discussions entre le curé et le conseil municipal ne sont pas rares.

L'unité de base de cette société est la famille. Chaque groupe familial forme une entité propre, ayant des liens économiques et affectifs très forts. Malgré tout, il ne faut pas croire que chaque famille soit refermée sur elle-même. Au contraire. Deux facteurs favorisent les rapports inter-familiaux. En premier lieu, la parenté est très étendue. On garde contact avec les cousins du quatrième et même du cinquième degré. A l'occasion des fêtes, des baptêmes, des mariages, on se visite entre parents. Ce sont les vieillards qui servent de "démêleurs de parenté". Quand quelqu'un n'est pas sûr des liens exacts qui le rattachent à telle ou telle personne, il va consulter l'un des anciens du village.

En second lieu, les membres de la communauté se rencontrent à l'occasion des veillées et autres manifestations collectives. A ce moment, tout le monde fraternise ensemble et il n'y a pratiquement pas de barrières familiales ou parentales. Un exemple suffira. Dans les veillées, tous les enfants jouent ensemble. Si l'un d'eux cause du trouble, n'importe quel adulte, parent ou non, a le droit de le punir (en général, il le fait agenouiller dans un coin).

Mais la famille garde son rôle d'unité économique et affective. Nous allons maintenant en examiner la structure interne. En général, chaque groupe familial se compose du père, de la mère et de leurs nombreux enfants. Souvent aussi, on peut ajouter à cela les grands-parents. Les relations entre parents et enfants sont cordiales, mais assez sévères. L'obéissance doit être strictement observée. Quand on ordonne quelque chose à 
un enfant, on ne lui explique pas les raisons de cet ordre. Il doit obéir promptement, sans poser de questions. Quand les grandsparents demeurent chez leur fils, les relations entre eux et leur bru sont très bonnes. La femme les considère comme ses propres parents. Les frères et sœurs s'entendent bien. Ils considèrent comme sacré le lien qui les unit. Ce sont les parents qui enseignent à leurs enfants les travaux domestiques et ceux de la ferme, ainsi que le bornage, les limites des différentes propriétés de la localité.

Les aînés aident beaucoup leurs parents pour ce qui est de l'éducation de leurs jeunes frères. Ce sont eux qui s'en occupent, qui les amusent et les bercent. Jusqu'à l'âge de treize ans, l'enfant participe à l'entretien de la maison et de la ferme, mais il considère cela comme un jeu et un apprentissage plutôt que comme un travail. D'ailleurs, pendant cette période de sa vie, il faut qu'il s'instruise. Durant les quatre ou cinq ans passés sur les bancs de l'école, le temps consacré au travail manuel est réduit de beaucoup. Mais à partir de treize ans, tout le monde, garçons et filles, se consacrent aux tâches agricoles.

Quelques années plus tard, on arrive à l'âge des fréquentations. Les adultes organisent des veillées spéciales où l'on présente les garçons aux filles. Les jeunes gens auront par la suite bien d'autres occasions de se rencontrer. Dans les soirées et les fêtes communautaires, ils forment un groupe à part de celui des adultes. Quand un garçon a l'intention de se marier, il demande à une fille la permission de la courtiser. Si elle accepte, il va veiller chez elle deux ou trois fois par semaine. Cela dure de six mois à un an. Quand il juge le moment venu, le jeune homme fait la grande demande. Normalement, les fiançailles suivent quelque temps plus tard.

On se marie surtout entre gens de la même paroisse. Un soupirant qui va faire la cour à une jeune fille de la paroisse voisine risque de se faire casser la figure par les concitoyens de la demoiselle. Les mariages entre proches parents sont très peu nombreux. Il faut une dispense de l'Église et la plupart des 
cultivateurs la jugent trop onéreuse pour leurs moyens. De plus, on voit une telle union d'un très mauvais œil, car on craint que les époux aient des enfants infirmes.

Le nouveau couple s'installe généralement sur la terre des parents du marié. On peut expliquer cela par les règles d'héritage. La maison paternelle échoit normalement à l'aîné des garçons tandis que ses frères reçoivent une parcelle de la terre ancestrale (l'argent étant presque inexistant, on ne lègue que des biens en nature). L'aîné se trouve favorisé, mais il est dans l'obligation de rendre service à ses frères et sœurs en cas de besoin. Les filles n'héritent pas puisque par leur mariage elles vont vivre sur la terre de leur époux. Par contre, au moment des épousailles, le père leur fait don de deux moutons, de deux porcs et d'une vache à lait. Le futur mari acceptera avec plaisir cette dot, surtout si sa femme y ajoute un trousseau de noce qụ'elle a elle-même préparé.

\section{IV - FOLKLORE}

La vie traditionnelle est marquée par un grand nombre de coutumes, de croyances et de fêtes qui lui donnent une dimension spirituelle et viennent rompre la monotonie du travail quotidien. Nous allons examiner ici les principales occasions où cet aspect de l'existence apparaît au grand jour.

Les fêtes auxquelles on attache le plus d'importance sont celles du calendrier liturgique. Noël, le Jour de L'An et l'Épiphanie sont fêtés d'une manière toute spéciale. Il en est de même, quoique à un moindre degré, de Pâques, de la Fête-Dieu et de la Toussaint. De plus, certaines dates sont marquées par des réjouissances qui ne se rattachent pas directement au mystère religieux du jour. C'est le cas du Mardi-Gras et de la SaintJean. Dans les paragraphes qui suivent, nous allons parcourir le cycle annuel des fêtes en décrivant sommairement les coutumes rattachées à chacune d'elles.

Au matin du Jour de l'An, on se lève à quatre heures. Pendant que les plus jeunes s'habillent, les hommes de la maison allument un feu à l'extérieur afin de saluer les parents et les 
amis. Puis tout le monde s'en va chez le grand-père paternel. Vers six heures, les enfants et les petits-enfants rassemblés autour de l'aïeul reçoivent sa bénédiction. Après avoir pris le traditionnel verre de vin du Jour de l'An, on déjeune et on se rend à la grand'messe. Puis c'est le dîner de famille. Tous les parents se serrent la main et s'embrassent en s'offrant mutuellement leurs meilleurs vœux. A table, on laisse une place vide appelée l'assiette du pauvre pour que si quelque étranger se présente, il puisse avoir part aux réjouissances. Pendant tout l'après-midi et toute la nuit, on danse, on chante et on se fait des étrennes. Ce n'est qu'au petit matin que chacun rentre chez soi.

Le jour des Rois, c'est un peu la même chose qui recommence. Après la messe, tout le monde se rassemble pour danser. La fête dure encore toute la nuit. Au dîner, on mange le gâteau des Rois. Ceux qui trouvent le pois et la fève sont couronnés roi et reine de la journée. Ils doivent s'embrasser et ouvrir le bal. Pendant la période qui s'étend de Noël au Mardi-Gras, presque chaque soir est un soir de veillée. Comme le travail de la ferme est presque réduit à rien, on en profite pour s'amuser.

Le soir du Mardi-Gras, plusieurs personnes se déguisent et vont de porte en porte. On essaie de découvrir leur identité. Dans beaucoup de maisons il y a une veillée et les masques participent à chacune d'elles pendant quelques minutes. Ils terminent la soirée à la dernière porte où ils frappent. A minuit, le carême commence. Toutes les réjouissances s'arrêtent brusquement. A part quelques petites veillées (et quelques déguisements) à la mi-carême, elles ne recommenceront qu'à Pâques. Le printemps est la saison du sucre d'érable. Au mois d'avril, on se rassemble à la cabane à sucre le dimanche après-midi pour manger des crêpes et de la "trempette".

Le matin de la Résurrection, il faut sortir du lit avant le lever du soleil pour aller chercher l'eau de Pâques. Après l'avoir puisée dans un ruisseau d'eau vive, on en boit un verre. Cela gardera en santé pour le reste de l'année. Le père enlève un des chassis-doubles de la cuisine pour que la "Bonne femme 
Pâques" puisse venir déposer des œufs en sucre d'érable sur le rebord de la fenêtre. A la fin de l'après-midi, une bonne partie de la parenté se réunit pour le souper de famille. Après cela, on danse et on chante jusque tard dans la soirée.

Aux Rogations, les cultivateurs font bénir leurs semences. Les graines ainsi consacrées seront plantées les premières. A la même occasion, on organise une procession pour bénir les champs. Tout au long de l'année d'ailleurs, il peut y avoir des processions spéciales pour demander de la pluie, pour la faire cesser ou encore pour enrayer une épidémie. Au mois de mai, la famille se rassemble tous les soirs au pied de la croix du chemin pour chanter des cantiques et réciter des prières. Le jour de la Fête-Dieu, le Saint Sacrement parcourt le village précédé de bannières et d'enfants qui jettent des fleurs sur son passage. A plusieurs endroits, on a érigé des reposoirs. La procession arrête pendant quelques minutes à chacun d'entre eux.

La Saint-Jean-Baptiste est avec le temps des Fêtes et le Mardi-Gras l'une des grandes occasions de réjouissances de l'année. Le soir du 23 juin, on allume un grand feu de joie en face de l'église. Au même moment, on voit des lueurs apparaître sur l'île d'Orléans. Ce sont les insulaires qui répondent à leurs amis de la côte de Beaupré. La nuit se passe à danser autour du brasier et à s'envoyer des messages lumineux. Remarquons que dans les "Anciens Canadiens" Philippe-Aubert de Gaspé signale une pratique semblable à la fin du XVIII ${ }^{e}$ siècle.

L'été et l'automne sont moins riches en fêtes que le reste de l'année. C'est l'époque où les travaux agricoles battent leur plein. On n'a pas le temps de chômer. Au mois d'août, on organise quelques épluchettes de blé d'Inde mais à part cela, il n'y a pas de grandes réjouissances jusqu'à Noël. A la Toussaint, c'est la "vente des Âmes", sorte d'encan dont les profits serviront à faire dire des messes pour les défunts. Le 25 novembre, on fabrique de la tire à l'occasion de la Sainte-Catherine.

La veille de Noël, après avoir dressé le traditionnel sapin, on se rend à l'église vers dix heures trente du soir. Après la confession, on assiste à la messe de minuit et de retour à la 
maison, on réveillonne et on danse. Les plus jeunes trouvent dans leur bas les bonbons que l'Enfant Jésus leur a apportés. L'avant-midi du jour de Noël est consacré aux visites de famille puis, après le dîner, on retourne à l'église pour la bénédiction des enfants et celle des gorges (pour laquelle on invoque saint Blaise). La soirée se passe tranquillement à jaser entre amis et à chanter un peu. Quelques jours plus tard, c'est la guignolée. Un groupe d'hommes va de porte en porte en amassant des dons pour les pauvres de la paroisse.

En dehors des fêtes qui reviennent annuellement, on trouve à se réjouir à l'occasion des baptêmes, des mariages et même des funérailles. Si la première de ces cérémonies passe presque inaperçue, ce n'est pas le cas des deux autres; commençons par le mariage. En général, celui-ci a lieu le lundi. Depuis plusieurs mois, les deux jeunes gens se sont fébrilement préparés à ce jour. La fiancée a fabriqué elle-même la robe qu'elle portera à cette occasion. Le matin des noces, c'est sa marraine qui l'aide à s'en revêtir. Puis le jeune homme vient chercher sa future épouse pour la mener à l'église. Après la cérémonie religieuse, c'est le festin qui commence. Tout le village est invité (la plupart des gens fournissent eux-mêmes leur nourriture). On dresse de grandes tables en plein air et pendant trois jours, on mange et on danse presque sans arrêt. Notons que pendant la période des fiançailles, le jeune homme n'a pas le droit de coucher dans la même maison que sa fiancée. Cette coutume est exactement contraire à celle qui a cours dans de nombreuses parties de la France et qui veut que le futur époux passe au moins une nuit sous le même toit que sa promise.

Venons-en maintenant aux funérailles. Quand une personne meurt, on l'étend sur des planches de bois et on la recouvre d'un suaire. Pendant trois jours et trois nuits, hommes et femmes veillent son corps. D'heure en heure, on récite le chapelet. Entretemps, les hommes boivent et se racontent des histoires d'épouvante (surtout la nuit). Le matin des funérailles, on met le cadavre dans un cercueil sur lequel on a cloué une plaque de métal portant le nom du défunt. Puis le cortège se met en marche 
vers l'église. La tombe est précédée par une grande croix noire (de tempérance). Parents et amis suivent en arrière. Après les funérailles, on se rend au cimetière. Le cercueil est descendu dans la fosse et avant de partir, les proches parents du défunt jettent une poignée de terre dans celle-ci.

Le folklore d'une communauté traditionnelle ne s'exprime pas seulement dans les fêtes et les cérémonies, mais aussi à travers les croyances et les légendes. Nous traiterons brièvement de ces deux derniers modes d'expression de l'âme populaire.

Sur la côte de Beaupré comme partout ailleurs, on connaît un bon nombre de moyens de guérir les maladies. Évidemment, dans les cas graves, on a recours au docteur, mais pour ce qui est des troubles courants, on se fie aux recettes de la médecine populaire. Par exemple, l'herbe à dinde et l'herbe à chat sont employées couramment, ainsi que le sirop blanc. Pour guérir une blessure, on boit une tisane faite d'écorce de cormier rouge bouillie. Si la plaie s'envenime, il faut utiliser le plantain. Il est aussi recommandé de prendre du soufre dans du sirop. En cas de fêlure ou de fracture, on va voir le "rammancheur". Si une personne ne parvient pas à se guérir au moyen des remèdes, elle peut demander l'aide de quelqu'un qui a un don. En récitant la formule appropriée, celui-ci pourra "enlever le feu" des brûlures ou faire partir un clou. Pour que son intervention soit efficace, il ne faut pas le remercier. On croit généralement que dans une famille où il $\mathrm{y}$ a sept garçons ou sept filles de suite, le septième a le don de guérir toutes les maladies.

Les gens ont également une grande confiance dans le pouvoir de Dieu et des saints. Par exemple, l'image de la Sainte Face fixée au mur d'une maison la préservera de l'incendie. Mais le monde n'est pas seulement peuplé d'êtres qui veulent le bien de l'humanité. Au contraire. On craint beaucoup les "jeteux de sorts". Ceux-ci peuvent causer la maladie ou la mort par un simple effort de leur volonté. Mais il y a un moyen de les démasquer. Quand un animal meurt à la suite d'un sort, on découpe sur lui un quartier de viande et on le brûle. Celui qui a jeté le sort ressent cette brûlure et pour en être débarrassé, 
il doit avouer son forfait. On raconte à ce sujet qu'une vache étant morte d'une manière suspecte, le propriétaire de l'animal alla brûler la carcasse au bord du fleuve. Après quelques minutes, on vit une barque se détacher de l'île d'Orléans et se diriger vers la côte. Dès qu'il fut à portée de voix, celui qui montait l'embarcation cria que c'était lui le coupable, qu'il souffrait beaucoup et qu'il promettait de ne jamais recommencer. On éteignit le feu et le "jeteux de sort" fut immédiatement soulagé.

Celui qui ne fait pas ses Pâques pendant sept ans risque de "courir le loup-garou". On cite le cas d'un homme qui se transformait en bœuf à tous les soirs. S'il n'est pas changé en animal, le mécréant peut être condamné à errer sous la forme d'un feu-follet. Parfois, cette métamorphose a lieu du vivant de l'individu. C'est ce qui arriva à un bûcheron de la région. Chaque soir, après le souper, il quittait le réfectoire du chantier sans dire un mot. Un jour, deux de ses compagnons partirent à sa recherche. Au milieu d'une clairière, ils trouvèrent son corps inanimé, la bouche ouverte. L'âme du bûcheron était sortie par cet orifice et voletait autour de sa tête sous la forme d'une boule de feu (c'est une croyance générale que l'âme quitte le corps par la bouche).

Autrefois, certains damnés purgeaient leur peine sur la terre. Ici encore, ils pouvaient le faire sous la forme de feuxfollets. Un citoyen de l'Ange-Gardien, voyant une grosse boule de feu devant sa maison, planta son couteau sur un piquet de clôture (c'est le geste rituel à faire pour délivrer l'âme emprisonnée dans la flamme). Il ne s'était pas plus tôt retourné pour rentrer chez lui qu'il entendit le couteau siffler à ses oreilles et aller s'enfoncer dans le cadre de la porte. Notre homme se promit bien de ne plus jamais venir en aide à un feu-follet en peine.

Partout dans le monde, il existe des légendes où le surnaturel se mêle à la vie quotidienne. La côte de Beaupré ne fait pas exception à cette règle et les récits fantastiques y sont aussi nombreux qu'ailleurs. Nous nous contenterons de citer trois d'entre eux. 
La légende la plus connue est celle de la Dame Blanche. Sous le régime français, un soldat partit pour la guerre. Sa fiancée, après l'avoir attendu de longues années, s'imagina qu'il ne reviendrait jamais et, de désespoir, se jeta dans la chute Montmorency. Depuis cette époque, elle revient régulièrement hanter le lieu de son suicide. Mais les apparitions du surnaturel peuvent être plus concrètes, plus proches de nous. A l'AngeGardien, on peut voir une maison qui a déjà reçu la visite du diable. Au cours d'une veillée, un bel étranger se présenta pour danser. Tout le monde lui fit bon accueil, sauf la vieille grand'mère qui disait son chapelet. Elle avait remarqué une lueur étrange dans les yeux de cet homme. La soirée était assez avancée quand soudain, la jeune fille qui dansait avec l'étranger poussa un cri. Malgré la paire de gants qu'il portait, celui-ci l'avait égratignée. Elle comprit que son partenaire n'était nul autre que Lucifer et que c'était des griffes qu'il cachait sous ses gants. Aussitôt, elie fit son signe de croix et le dẻmon disparut en laissant la trace de ses pas devant la maison. Notons que le thème du "diable beau danseur" est très répandu dans les légendes canadiennes.

On raconte enfin qu'à Sainte-Anne-de-Beaupré on voulait installer dans la nouvelle église une vieille statue de la sainte qui se trouvait dans l'ancienne chapelle. On procéda au déménagement, mais le lendemain, la statue avait miraculeusement regagné son emplacement original. Malgré tous les efforts déployés, on ne réussit pas à la garder dans le temple neuf et on finit par la laisser où elle voulait être.

En général, les gens ont foi dans toutes ces croyances et légendes mais cela ne les empêche pas d'être très réalistes. Ils connaissent la limite entre imagination et réalité. Même s'ils admettent que le surnaturel puisse intervenir dans toutes les actions de l'existence, ils gardent leur sens pratique et accordent la priorité aux qualités acquises et au travail concret.

LOUIS-JACQUES DORAIS

Janvier 1965. 